\title{
Análisis de las temporadas de veda en la explotación marina de camarones del Golfo de Tehuantepec, México
}

\author{
Analysis of the closed seasons in the marine shrimp exploitation of the Gulf of Tehuantepec, Mexico
Pedro Cervantes-Hernández ${ }^{*}$, María Isabel Gallardo-Berumen', Sebastián Ramos- Cruz $^{2}$, Mario Alejandro Gómez-Ponce ${ }^{3}$ y Adolfo Gracia Gasca ${ }^{3}$

\author{
${ }^{1}$ Instituto de Recursos, Universidad del Mar - Puerto Ángel, A. P. 47, C.P. 70902, Puerto Ángel, Oaxaca, México. \\ *Alumno adscrito al posgrado del Instituto de Ciencias del Mar y Limnología, Universidad Nacional Autónoma de México, México \\ ${ }^{2}$ Programa Camarón del Instituto Nacional de la Pesca, Centro Regional de Investigación Pesquera - Salina Cruz. \\ C.P. 70680, Salina Cruz, Oaxaca, México \\ ${ }^{3}$ Laboratorio de Ecología Pesquera de Crustáceos, Instituto de Ciencias del Mar y Limnología, Universidad Nacional Autónoma \\ de México. A. P. 70-305; 04510, México, D.F. \\ pch@angel.umar.mx
}

\begin{abstract}
The brown shrimp (Farfantepenaeus californiensis) and white shrimp (Litopenaeus vannamei) fishery exploitation in the Gulf of the Tehuantepec (GT) between 1993 and 1997 is described. Two population indices were obtained for each species: the recruits abundance index (RA), and the spawners abundance index (SA). Five closed seasons (from May/June to August) were considered in order to analyze the annual variation of these indices based on two spectral components: the cycle and the tendency. The first component indicated that the closed seasons protected almost $100 \%$ of the white shrimp recruits and $90 \%$ of the brown shrimp recruits. The brown shrimp fishery resulted in a good exploitation state because the abundance tendency of the recruits and spawners were stable or constant. The white shrimp fishery resulted in an overfished state, because the abundance tendency of the recruits and spawners were in decline. The closed seasons suitably do not fulfil the objectives for what was implemented, that is, the simultaneous protection of the periods of reproduction and egg-laying in the high sea fishing zones. At the moment, these are applied from March to September, whereas the shrimp production continuously descends in the GT. A change to these dates from June/July to October was proposed to assure the protection of the spawners and the growth of the recruits of both species. Additionally, it is necessary to regulate the lagoons systems fisheries in the GT to optimize the production in the lagoon and of high sea.
\end{abstract}

Key words: Evaluation, brown shrimp, white shrimp, abundance indices

\section{Introducción}

La pesca de camarón en el Pacífico mexicano (Pm en adelante), incide sobre de cuatro especies de interés comercial: Farfantepenaeus californiensis (Holmes, 1900) o camarón café, Litopenaeus vannamei (Boon,
Resumen.- Se describe el estado de explotación de las pesquerías de camarón café (Farfantepenaeus californiensis) y camarón blanco (Litopenaeus vannamei) en el Golfo del Tehuantepec (GT), entre 1993 y 1997. Para cada especie, se obtuvieron dos índices poblacionales: la abundancia de reclutas (FR) y la abundancia de reproductores (DA). Cinco temporadas de veda (de mayo/junio a agosto) fueron consideradas para analizar la variación anual de estos índices mediante dos componentes espectrales: el ciclo y la tendencia. La primera componente indicó que las vedas protegieron cerca del 100\% de los reclutas de camarón blanco y 90\% de camarón café. Esta última pesquería se observó en un estado aceptable de explotación, ya que la tendencia de la abundancia de reclutas y reproductores fue estable. La pesquería de camarón blanco se observó sobre-explotada, debido a que la tendencia de la abundancia de reclutas y reproductores fue descendente. Las temporadas de veda no cumplen adecuadamente con los objetivos para los cuales fueron implementadas, esto es, la protección simultánea de los períodos de reproducción y de desove en las zonas de pesca de alta mar. Actualmente, éstas se aplican de marzo a septiembre, en tanto que la producción de camarón continua al descenso en el GT. Se propone un corrimiento a dichas fechas, de junio/julio a octubre, para asegurar la protección de los reproductores y el crecimiento de los reclutas de ambas especies. Adicionalmente, es necesario reglamentar la pesca en los sistemas lagunares del GT, para optimizar la producción ribereña y de alta mar.

Palabras clave: Evaluación, camarón café, camarón blanco, índices de abundancia
1931) o camarón blanco, Farfantepenaeus brevirostris (Kingsley, 1878) o camarón cristal y Litopenaeus stylirostris (Stimpson, 1871) o camarón azul (CervantesHernández 2008). Esta pesquería se desarrolla desde la zona del Alto Golfo de California, México (en el delta del Río Colorado), hasta la frontera con Guatemala e 
incluye la explotación ribereña de camarón en la etapa juvenil en todos los sistemas lagunares del Pm.

La explotación de camarón en el Pm, ha sido regulada mediante un sistema unificado de vedas temporales, cuyos objetivos han sido y siguen siendo, la protección simultánea de los períodos de reproducción y de desove en las zonas de pesca de alta mar (INP 1997-1998). Debido a la diferencia de producción que existe entre las zonas norte de pesca (Zona 10: El Sauzal-Baja California Norte, 50: La Paz-Baja California Sur, 20: Guaymas, Sonora, 30: Mazatlán, 40: Sinaloa y 60: Bahía de Banderas, Nayarit) y la sur (Zona 90: Salina Cruz, Oaxaca), ésta última con menor producción que las primeras. La fecha de inicio y finalización de las vedas temporales, se han modificado desde su establecimiento en 1938, dependiendo en gran medida, de los resultados históricos y las prospecciones pesqueras que se han generado en las zonas norte de pesca. De manera que, entre 1980 y 1987, las vedas se establecieron de julio a septiembre (López-Martínez et al. 2001), en 1993 fueron instituidas entre mayo/junio y septiembre, en 1999 de abril a mayo y actualmente de marzo a septiembre (INP 2007a, 2007b).

En el Golfo de Tehuantepec (GT en adelante), las embarcaciones comerciales operan con redes de arrastre de 57,15 mm de luz de malla, entre las cinco y 40 brazas de profundidad (9,1 y 72,8 m) (INP 2004). La captura industrial de camarón se realiza en el ambiente marino en la Zona 90 de pesca, ubicada entre Punta Chipehua en Salina Cruz, Oaxaca $\left(16^{\circ} 10^{\prime} \mathrm{N}\right.$ y $\left.95^{\circ} 11^{\prime} \mathrm{W}\right)$ y Puerto Chiapas, Chiapas $\left(14^{\circ} 41^{\prime} \mathrm{N}\right.$ y $92^{\circ} 24^{\prime} \mathrm{W}$ ) (Fig. 1). A lo largo de la línea costera, se localiza un gran número de marismas y sistemas lagunares (La Joya-Buenavista, Los Patos-Solo Dios, Carretas-Pereyra, Chantuto-Panzacola), de las que sobresalen por sus dimensiones el Sistema Lagunar Huave en Oaxaca y el Mar Muerto compartido por los estados de Oaxaca y Chiapas (Reyna-Cabrera \& Ramos-Cruz 1998) (Fig. 1), en las cuales, se lleva a cabo la explotación ribereña de camarón en la etapa juvenil, principalmente sobre L. vannamei (Cervantes-Hernández et al. 2006).

Después de décadas de aplicar el sistema unificado de vedas temporales y sus modificaciones en todo el Pm, Cervantes-Hernández et al. (2006) reportaron que la presión pesquera sobre las poblaciones de camarón del GT, se ha incrementado en alta mar y en los sistemas lagunares entre 1993 y 2002, por ello, es pertinente revisar y hacer una evaluación acerca de su efectividad en el GT, en donde el recurso camarón está sujeto a una pesca secuencial, dentro de un ambiente que exhibe características netamente tropicales y patrones de reclutamiento y de reproducción, más continuos y acentuados que las zonas de pesca subtropicales del norte del Pm.

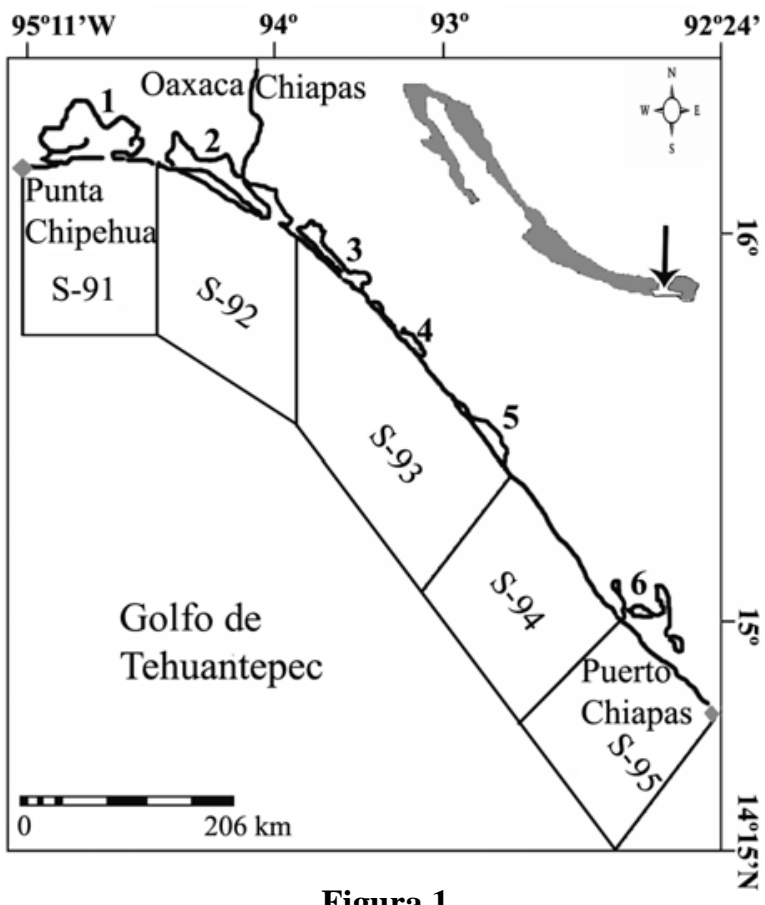

Figura 1

Localización geográfica de la zona de pesca 90 en el Golfo de Tehuantepec. S-91 a S-95 son sub-sectores. (1) Sistema Lagunar Huave, (2) Laguna Mar Muerto, (3) La Joya Buenavista, (4) Los Patos-Solo Dios, (5) Carretas Pereyra y (6) Chantuto-Panzacola

Geographic location of fishing zone 90 in the Gulf of Tehuantepec. S-91 to S-95 are sub-sectors. (1) Sistema Lagunar Huave, (2) Laguna Mar Muerto, (3) La Joya Buenavista, (4) Los Patos-Solo Dios, (5) Carretas Pereyra and (6) Chantuto-Panzacola

En este trabajo se analiza el estado de explotación de las poblaciones de F. californiensis y L. vannamei del GT, México, con base en el efecto que tuvieron entre 1993 y 1997, cinco temporadas de veda y cuatro de pesca, sobre la variación anual de la abundancia de los reclutas y los reproductores de ambas especies.

\section{Material y métodos}

Para el análisis poblacional de $F$. californiensis y $L$. vannamei, se utilizó la captura comercial clasificada por categorías (10-14, 15-20, 21-25, 26-30, 31-35, 36-40, 4150, 51-60 y 60 abdómenes por libra de peso o 0,453 Kg), extraída de la Zona 90 de pesca y registrada entre 1993 y 1997, en los puertos de Salina Cruz, Oaxaca y Puerto Chiapas, Chiapas. Esta información fue proporcionada a la Universidad del Mar en Puerto Ángel, Oaxaca, México, por el Centro Regional de Investigación Pesquera de Salina Cruz, Oaxaca, mediante el proyecto CRIPUMAR:2IR043. 
Cinco temporadas de veda (mayo-agosto 1993, junioagosto 1994, 1995, 1996 y 1997) y cuatro temporadas de pesca (septiembre a mayo 93-94, 94-95, 95-96 y 96-97) fueron identificadas en los registros de la captura comercial. Las temporadas de veda aquí analizadas, presentaron variantes con relación a las fechas estipuladas en la bibliografía (INP 1997-1998), pero fueron consistentes con las fechas reportadas por INP (2007a, 2007b).

La captura comercial clasificada por categorías y la técnica propuesta por Gracia (1991), se utilizaron para obtener el número total de cohortes y el número total de camarones capturados mensualmente por grupos de edad. La técnica se adaptó para F. californiensis y L. vannamei considerando sexos combinados, ya que la información por sexos no estuvo disponible en los datos de la captura comercial.

El proceso fue el siguiente:

1.- Bajo el supuesto de que los registros del peso de los abdómenes dentro de cada una de las categorías se distribuye normalmente $\mathrm{Z}(0,1)$, se estimó la probabilidad del peso de cada abdomen $\mathrm{P}(\mathrm{a})$, usando el promedio y la desviación estándar que resultó en cada una de las categorías.

2.- Para cada categoría, la división del peso total entre su correspondiente sumatoria de $\mathrm{P}(\mathrm{a})$, generó un factor de conversión. Este factor fue multiplicado por $\mathrm{P}(\mathrm{a}) \mathrm{y}$ dividido entre la frecuencia de cada abdomen, para obtener el número total de camarones capturados por unidad de peso abdominal.

3.- Para obtener una estimación aproximada de la edad de los camarones capturados por unidad de peso abdominal, en cada una de las categorías, se emplearon las constantes del crecimiento en talla y peso reportados por Chávez (1979) para: F. californiensis: $\left(L_{\infty}=242 \mathrm{~mm}\right.$, $\mathrm{t}_{\mathrm{o}}=-0,344, \mathrm{k}=0,186$ y $\left.W_{\infty}=220 \mathrm{~g}\right)$ y L. vannamei: $\left(L_{\infty}=\right.$ $\left.200 \mathrm{~mm}, \mathrm{t}_{\mathrm{o}}=-0,293, \mathrm{k}=0,226 \mathrm{y} W_{\infty}=103 \mathrm{~g}\right)$.

Con base en el proceso anterior y para cada especie, se obtuvo una matriz con la estimación del número total de camarones capturados mensualmente por grupos de edad. Éstas y las tasas de mortalidad natural (M) estimadas por Ramos-Cruz et al. (2006) en la fase marina de cada especie, de 1989 a 1998 (M = 0,22 mensual, para camarón café y $\mathrm{M}=0$,20 mensual, para camarón blanco), se utilizaron para desarrollar el análisis de población virtual (APV) según Pope (1972), calibrado de acuerdo con Seber (1982).

Del APV se obtuvieron por separado dos matrices de abundancia virtual por grupos de edad, éstas son: $\left(\mathrm{X}_{\mathrm{APV} \mathrm{C}}\right)$ para camarón café y $\left(\mathrm{X}_{\mathrm{APVv}}\right)$ para camarón blanco. La abundancia virtual representa el número total de camarones, que debió existir mensual e independientemente, en cada una de las cohortes que conformaron la población natural de F. californiensis y L. vannamei.

Con base en el método propuesto por CervantesHernández (2008), la abundancia virtual se utilizó para obtener dos índices poblacionales por especie: a) la abundancia de reclutas (FR) (FR para camarón café y $\mathrm{FR}_{\mathrm{v}}$ para camarón blanco) y b) la abundancia de reproductores (DA) (DA para camarón café y DA para camarón blanco).

La variación anual de la abundancia de reclutas, se estructuró utilizando a todas las cohortes del grupo de cuatro meses (edad de reclutamiento); mientras que, la abundancia de reproductores, se obtuvo mediante la adición de todas las cohortes pertenecientes a los grupos de siete a 16 meses de edad. Lo anterior, se representó matemáticamente mediante las siguientes funciones:

$$
\begin{aligned}
& F_{c}=F_{v}=E_{4 r} \\
& D A_{c}=D_{v}=E_{7 r}+E_{8 r}+E_{9 r}+E_{10 r}+E_{11 r}+E_{12 r}+E_{13 r}+E_{14 r}+E_{15 r}+E_{16 r}
\end{aligned}
$$

Donde:

$\mathrm{FR}_{\mathrm{c}}$ y FR $\mathrm{v}$ son los índices de la abundancia de reclutas de camarón café y blanco, respectivamente.

$\mathrm{DA}_{\mathrm{c}} \mathrm{y}_{\mathrm{DA}}$ son los índices de abundancia de reproductores de camarón café y blanco, respectivamente.

$\mathrm{E}_{\mathrm{qr}}$ es la abundancia virtual del grupo de edad q en el mes r.

No se consideró la interacción de las poblaciones de F. californiensis y L. vannamei, ni éstas con respecto a otras poblaciones naturales en el ambiente marino del GT; sin embargo, el efecto de M en el APV, incluye las muertes ocasionadas por la depredación, la edad avanzada y las enfermedades (mortalidad denso-dependiente), además de las ocasionadas por la variabilidad ambiental (mortalidad denso-independiente). La tasa M se consideró constante durante el periodo de explotación que se ejerció sobre cada una de las cohortes.

La variación anual de la abundancia de los índices FR y DA para cada especie, se representó mediante series de tiempo entre 1993 y 1997. A cada serie se le aplicó el modelo de descomposición espectral de Kendall (1984), para obtener las siguientes series de tiempo modificadas o componentes espectrales: a) el ciclo (C) y b) la tendencia (T).

La componente C se utilizó para transformar la variación anual de la abundancia con escala ordinaria 
(entre cero y el máximo), a otra con escala de intervalo (con el valor de cero como punto medio); en la cual, la diferencia entre un determinado valor mensual y el promedio anual de la abundancia, se representó y ajustó dentro de un rango simétrico por encima (anomalía positiva) y por debajo (anomalía negativa) del valor cero. Con base en lo anterior, fueron resaltados con mayor exactitud, independientemente de la magnitud, las fechas de ocurrencia y el intervalo de tiempo que transcurre entre los máximos y mínimos de la abundancia de reclutas y reproductores en ambas especies. Una anomalía positiva, indica que la abundancia de cualquier índice poblacional estuvo disponible a la pesca y viceversa en el caso una anomalía negativa.

Al resultado gráfico que se obtuvo para la componente C, le fueron acoplados cinco rectángulos verticales, para representar la duración de cada una de las temporadas de veda. Los espacios entre dichos rectángulos, representan la duración de las cuatro temporadas de pesca.

La componente $\mathrm{T}$ se utilizó para suavizar la variabilidad mensual que constituye una serie anual de abundancia, con el objeto de resaltar la tendencia final en cada índice poblacional (tendencia ascendente, descendente o estable), después de haber ocurrido las temporadas de veda y pesca.

Las componentes C y T se obtuvieron para cada uno de los índices según Uriel (1995); el proceso, en términos de $Y_{i k}$ (i año, k estación del año) con m = 12 meses, fue el siguiente:

1.- Se ajustó una recta por mínimos cuadrados a las medias anuales de la abundancia. $\left(\bar{Y}_{i}\right)$

2.- Se estimaron las medias mensuales para los diferentes años

$$
\left(\bar{Y}_{. k}\right)
$$

3.- Se aisló la componente $\mathrm{T}$ con base en la serie de medias mensuales corregidas

$$
\bar{Y}_{. k}^{\prime}=-\frac{b(k-1)}{m}
$$

donde b es un coeficiente que se estimó para cada m

4.- Se calculó la media global corregida

$$
\bar{Y}^{\prime}=\frac{\bar{Y}_{.1}^{\prime}+\bar{Y}_{.2}^{\prime}+\ldots+\bar{Y}_{. m}^{\prime}}{m}
$$

5.- La componente T se estimó con:

$$
T_{i k}=\bar{Y}_{. k}^{\prime}-\bar{Y}^{\prime}
$$

y C con

$$
C_{i k}=\bar{Y}_{i}-\bar{Y}_{. k}
$$

\section{Resultados}

A partir de la información de la captura comercial clasificada por categorías, se obtuvo un total de 60 cohortes para ambas especies, en cada caso, se registraron 14 grupos de edad entre los tres y 16 meses. La abundancia virtual de los índices poblacionales obtenidos con base en las matrices $\mathrm{X}_{\mathrm{APV} \mathrm{c}} \mathrm{y}_{\mathrm{APVv}}$, fue evaluada en número de camarones $\mathrm{x} 10^{4}$.

La variación anual de la abundancia de los índices poblacionales que resultó al aplicar la función 1, se muestra en las Figs. 2 y 3.

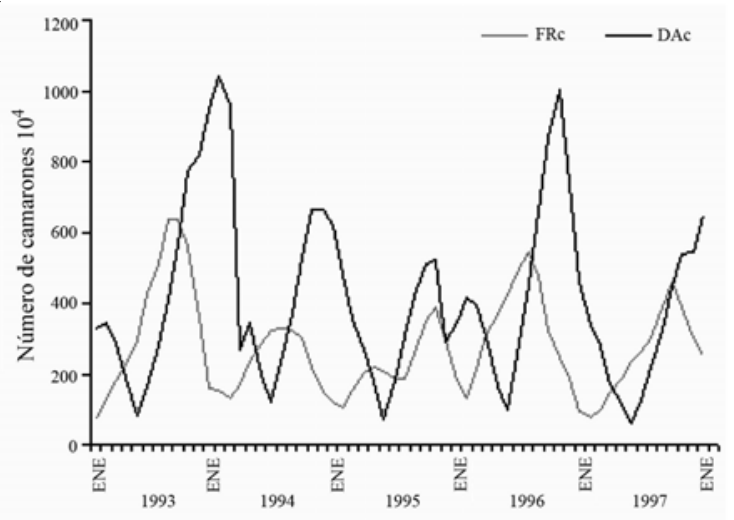

Figura 2

Variación anual de la abundancia de reclutas y reproductores de $\boldsymbol{F}$. californiensis en el Golfo de Tehuantepec de 1993 a 1997

Annual abundance variation of recruits and spawners of F. californiensis in the Gulf of Tehuantepec from 1993 to 1997

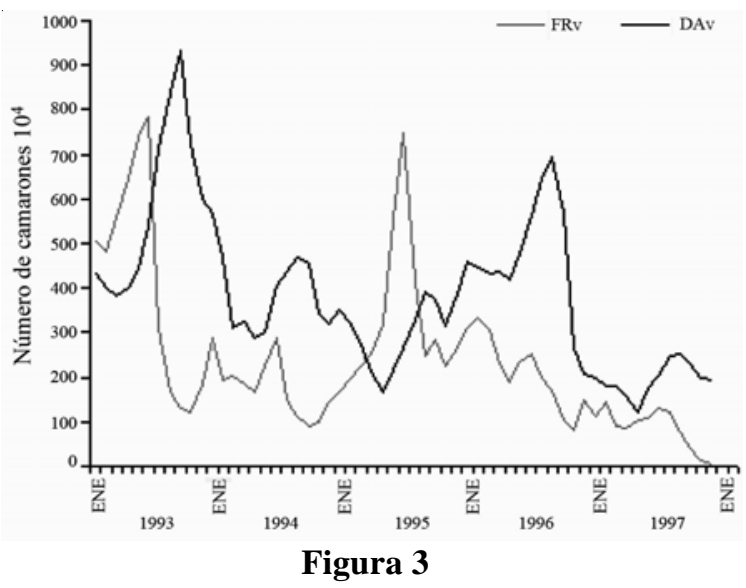

Variación anual de la abundancia de reclutas y reproductores de $L$. vannamei en el Golfo de Tehuantepec de 1993 a 1997

Annual abundance variation of recruits and spawners of $L$. vannamei in the Gulf of Tehuantepec from 1993 to 1997 
En la Fig. 2 se observó que los máximos de reclutamiento de camarón café (FR $)$, se presentaron en abril 1995, julio 1994 y 1996, agosto 1993, septiembre 1997 y octubre 1995. Los mínimos de este índice fueron observados entre enero y febrero de cada año. El índice DA fue máximo durante enero 1993 y 1998, febrero 1994, octubre 1993, 1995 y 1996, noviembre 1994. Los mínimos de este último índice, fueron observados entre mayo y julio de cada año (Fig. 2). Un efecto de correlación inversamente proporcional se obtuvo entre los índices FR y $\mathrm{DA}_{\mathrm{c}}(\mathrm{R}=-0,62, P=0,01)$, con un desfase aproximado de cuatro meses entre los respectivos máximos de abundancia. En el caso del reclutamiento de camarón blanco $\left(\mathrm{FR}_{\mathrm{v}}\right.$ ) (Fig. 3), los máximos se registraron en junio 1993, 1994, 1995, 1996 y 1997 y diciembre 1993, enero 1996 y 1997. Los mínimos de este índice fueron observados entre septiembre y octubre de cada año. Los máximos de reproductores $\left(\mathrm{DA}_{\mathrm{v}}\right)$ se observaron en enero 1993, septiembre 1993, agosto 1994, 1995, 1996 y 1997, diciembre 1994 y 1995. Los mínimos de este último índice ocurrieron entre marzo y abril de cada año (Fig. 3). Un efecto de correlación inversamente proporcional, se obtuvo entre los índices $\mathrm{FR}_{\mathrm{v}}$ y $\mathrm{DA}_{\mathrm{v}}(\mathrm{R}=-0,42, P=0,04)$, con un desfase aproximado de cuatro meses entre los respectivos máximos de abundancia.

Lo anterior fue analizado con mayor detalle para cada especie, utilizando las componentes C y T (Figs. 4 y 5).

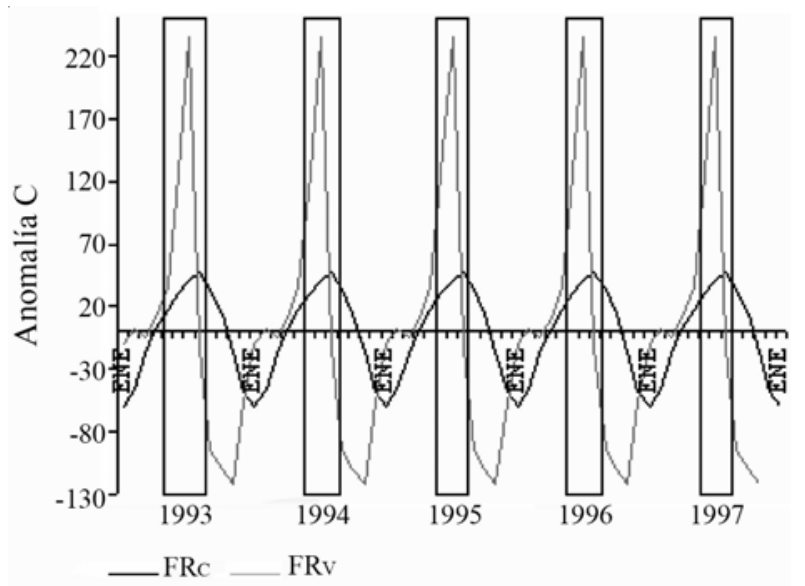

Figura 4

Variación anual de la componente $C$ para reclutas de $\boldsymbol{F}$. californiensis $\left(F_{\mathrm{c}}\right)$ y L. vannamei $\left(\mathrm{FR}_{\mathrm{v}}\right)$ en el Golfo de Tehuantepec de 1993 a 1997

Annual variation of component $C$ for recruits of $F$. californiensis (FRc) and L. vannamei (FRv) in the Gulf of Tehuantepec from 1993 to 1997

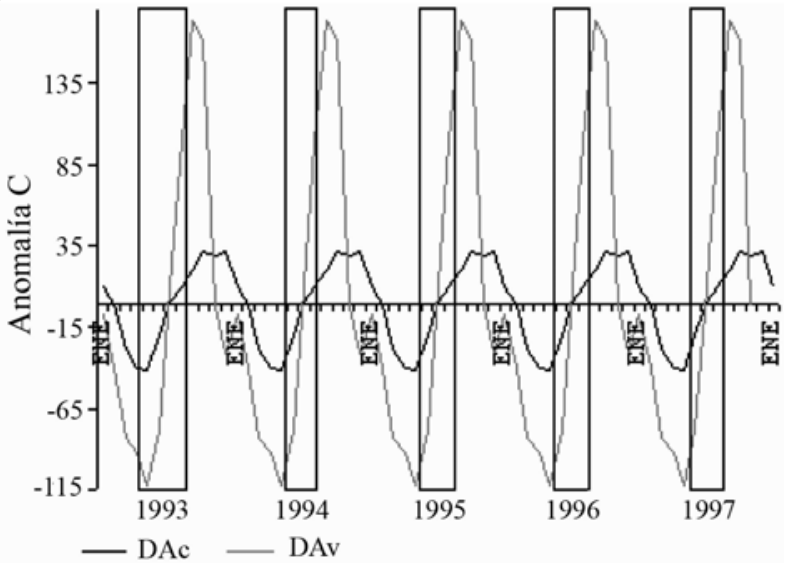

Figura 5

Variación anual de la componente $C$ para reproductores de F. californiensis (DA $)$ y L. vannamei $\left(\mathrm{DA}_{\mathrm{v}}\right)$ en el Golfo de Tehuantepec de 1993 a 1997

Annual variation of component $C$ for spawners of $F$. californiensis ( $\mathrm{DA}_{\mathrm{c}}$ ) and L. vannamei $\left(\mathrm{DA}_{\mathrm{v}}\right)$ in the Gulf of Tehuantepec from 1993 to1997

De acuerdo con la componente C, se observó que entre 1993 y 1997, las temporadas de veda protegieron cerca del $100 \%$ de los reclutas de camarón blanco, debido a que el intervalo de la anomalía positiva del índice $\mathrm{FR}_{\mathrm{v}}$ (de abril a agosto con máximos en junio), resultó menor en comparación con el intervalo observado para el índice $\mathrm{FR}_{\mathrm{c}}$ (de abril a octubre con máximos en agosto) (Fig. 4). Durante el transcurso de las temporadas de pesca, el índice $\mathrm{FR}_{\mathrm{v}}$ se mantuvo con signo negativo, sugiriendo con ello, que la edad de reclutamiento no estuvo disponible o fue capturada en menor proporción por la pesca de altura en el GT (Fig. 4).

Los reclutas de camarón café fueron protegidos en un $90 \%$, debido a una mayor amplitud de la componente C. La fracción restante fue capturada un mes al inicio (abril) y dos meses al final (entre septiembre y octubre) en el período de reclutamiento, durante las temporadas de pesca (Fig. 4). La anomalía FR cambió de signo (de positivo a negativo) al final del mes de octubre sugiriendo con ello, que la edad de reclutamiento, entre noviembre y marzo, no estuvo disponible o fue capturada en menor proporción, por la pesca de altura en el GT (Fig. 4).

De acuerdo con los resultados obtenidos se observó un desfase de tres meses entre los máximos $\mathrm{FR}_{\mathrm{v}}$ (junio) y $\mathrm{FR}_{\mathrm{c}}$ (agosto), al igual que entre los mínimos, registrados en noviembre y enero, respectivamente (Fig. 4).

$\mathrm{Al}$ igual que en el reclutamiento, el intervalo de la anomalía de los reproductores fue más amplia en el 
camarón café (de julio a febrero); mientras que, para el camarón blanco, éste se observó de junio a noviembre. En las temporadas de pesca, la componente $\mathrm{C}$ indicó que los reproductores de camarón blanco presentaron una disminución marcada durante los primeros meses (de septiembre a octubre) y a mediados de noviembre, se registró un cambio del signo (de positivo a negativo), el cual se mantuvo seis de los nueve meses que constituyen las temporadas de pesca (Fig. 5). Esta situación demuestra que los reproductores de camarón blanco no estuvieron disponibles o fueron capturados en menor proporción por la pesca de altura en el GT. Dentro del período de explotación, se observó una ligera recuperación del índice $\mathrm{DA}_{\mathrm{v}}$ (en enero); sin embargo, ésta no fue lo suficientemente fuerte como para revertir la anomalía negativa de la abundancia de reproductores (Fig. 5).

La anomalía positiva de los reproductores de camarón café se mantuvo el doble de tiempo con respecto a la del camarón blanco, lo que sugiere una mayor temporada de captura (de agosto a febrero); sin embargo, al finalizar el mes de febrero, la anomalía de abundancia DA ${ }_{c}$ evidenció un cambio de signo (de positivo a negativo), indicando un descenso en las capturas de los reproductores, que se extendió tres meses antes de concluir las temporadas de pesca (Fig. 5).

Los máximos $\mathrm{DA}_{\mathrm{v}}$ (septiembre) y $\mathrm{DA}_{\mathrm{c}}$ (octubre/ diciembre), presentaron un desfase de aproximadamente entre uno y tres meses; mientras que los mínimos fueron consistentes en mayo de cada año (Fig. 5).

Con base en lo anterior, el análisis de la componente C indicó que las temporadas de veda sólo protegieron una pequeña fracción de los reproductores de camarón café y camarón blanco (menos del 25\% en ambos casos) (Figs. 4 y 5). Por lo tanto, la protección de los reproductores en comparación con los reclutas de ambas especies fue relativamente baja durante el transcurso de estas temporadas.

En el camarón café, la componente $\mathrm{T}$ indicó que la abundancia del índice $\mathrm{FR}_{c}$ se mantuvo oscilando entre 62 y 185 x $10^{4}$ camarones; mientras que el índice DA se observó entre 40 y 122 x $10^{4}$ camarones (Fig. 6). Ambos índices no mostraron un patrón de cambio que confirmara una tendencia ascendente o descendente entre 1993 y 1997, por lo que la variación anual de la abundancia de éstos, resultó con una tendencia estable (Fig. 6).

En el camarón blanco, ambos índices poblacionales mostraron dos intervalos con tendencia descendente. En el primer intervalo, el índice $\mathrm{FR}_{\mathrm{v}}$ descendió de 547 a 159 x $10^{4}$ camarones entre mayo 1993 y octubre 1994. En el segundo intervalo, este mismo índice descendió entre

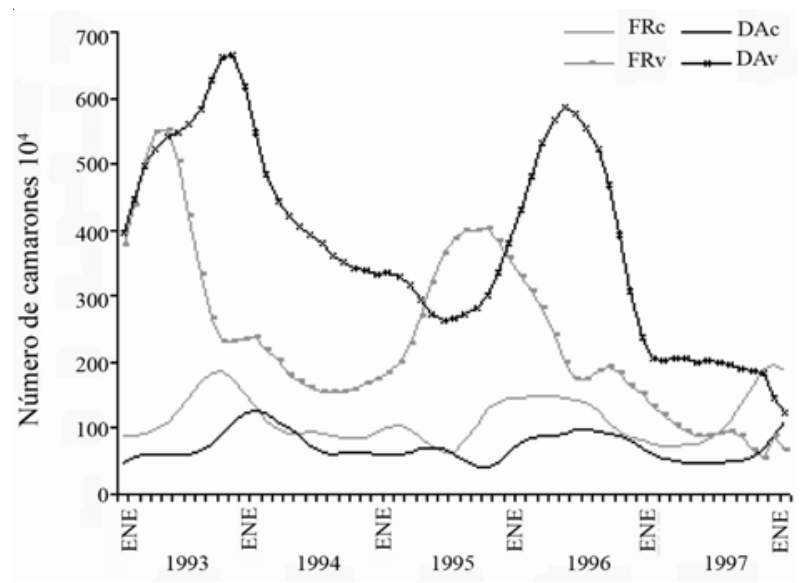

Figura 6

Tendencia de la abundancia de reclutas y reproductores de F. californiensis y L. vannamei en el Golfo de Tehuantepec de 1993 a 1997

Recruits and spawner abundance tendency of $F$. californiensis and L. vannamei in the Gulf of Tehuantepec from 1993 to 1997

octubre 1995 y enero 1997, de 401 a 60 x $10^{4}$ camarones (Fig. 6).

Con respecto al índice $\mathrm{DA}_{\mathrm{v}}$, los intervalos con tendencia descendente se registraron de noviembre 1993 a junio 1995, con un cambio de abundancia de 664 a 256 x $10^{4}$ camarones, respectivamente, y entre mayo $1996 \mathrm{a}$ enero 1997, con un cambio de abundancia de 584 a 122 x $10^{4}$ camarones, respectivamente (Fig. 6). Debido a lo anterior, la variación anual de la abundancia de ambos índices poblacionales, resultó con un patrón claramente descendente.

\section{Discusión}

$\mathrm{Al}$ igual que otras actividades pesqueras en México, la pesca de camarón se rige por la Ley de Pesca (Diario Oficial de la Federación 25/06/92) y la Norma Oficial Mexicana NOMPESC-002-1993 (Diario Oficial de la Federación 31/12/93). Sin embargo, esta normatividad no contempla una diferenciación en el manejo de las pesquerías de camarón del Golfo de Tehuantepec, con respecto al resto del Pacífico mexicano, el cual exhibe características netamente tropicales y patrones de reclutamiento y de reproducción más continuos y acentuados que las zonas de pesca subtropicales del norte del Pm, en donde las capturas de camarón son mayores.

Por décadas, las vedas temporales para camarón en el GT, se han establecido considerando únicamente los resultados históricos y las prospecciones pesqueras que 
se han generado en las zonas norte de pesca del Pm (Rodríguez de la Cruz 1976, Magallón-Barajas 1987, Morales-Bojórquez et al. 2001). Lo anterior ha sido ocasionado en parte, por el retraso histórico de las investigaciones pesqueras de camarón en la Zona 90 (Salina Cruz, Oaxaca) y en los sistemas lagunares del GT. Debido a lo anterior y por razones prácticas, las modificaciones referentes al inicio y finalización de las temporadas de veda y pesca, se ha generalizado a todo el $\mathrm{Pm}$, sin considerar adecuadamente la diferenciación entre especies, regiones, patrones de reclutamiento y de reproducción.

Los resultados obtenidos en este trabajo, sugieren que para el GT es conveniente una diferenciación en la aplicación de las temporadas de veda con respecto a todo el Pm. Lo anterior se fundamenta, en que durante el periodo en estudio, se protegió casi en su totalidad a los reclutas de camarón blanco (100\%) y camarón café (90\%); mientras que, sólo una fracción de los reproductores de ambas especies se protegió en dichas temporadas. Por otra parte, se observó que el efecto final de la aplicación de las cinco temporadas de veda en el GT entre 1993 y 1997, resultó en un estado aceptable de explotación para la pesquería de camarón café; por el contrario, la pesquería de camarón blanco mostró un estado de sobreexplotación, el cual se evidenció con las tendencias descendentes que presentaron los índices $\mathrm{FR}_{\mathrm{v}} \mathrm{y} \mathrm{DA}_{\mathrm{v}}$.

Los registros de producción durante el período 19831993, muestran que las capturas totales de camarón blanco (2,000-3,500 t), fueron mayores a las del camarón café (500-1,000 t) (INP 2000). Los resultados obtenidos con base en la componente T, sugieren que la abundancia de camarón blanco comenzó a descender de mayo 1993 a octubre 1994 y más drásticamente entre octubre 1995 y enero 1997; mientras que, la abundancia de los índices de camarón café aparentemente permanecieron estables. Como resultado ocurrió un cambio en la proporción de las capturas por especie con mayor magnitud para $F$. californiensis y menor para L. vannamei. Ramos-Cruz et al. (2006) reportan un comportamiento similar en las temporadas de pesca 1995-1996 y 1996-1997. Posteriormente, en las temporadas 2003-2004 y 20062007, se presentó un nuevo cambio en la composición de la captura por especies, que resultó en una proporción similar al de las temporadas comprendidas entre 19831993; no obstante, la captura total disminuyó a 945 t (INP, 2004) y a 429.4 t (INP, 2007a) en los últimos años. Esta tendencia sugiere un incremento sostenido del esfuerzo pesquero sobre las poblaciones de camarón del GT, en especial para el camarón blanco.

Parte de este incremento se debe a la alta presión de pesca que se ejerce sobre las etapas juveniles en los sistemas lagunares del GT, lo cual afecta negativamente a las poblaciones naturales de camarón (CervantesHernández et al. 2006). La pesca ribereña de camarón forma parte de la explotación secuencial que se ejerce sobre los camarones de la familia Peneidae, principalmente sobre los camarones del género Litopenaeus (Gracia 1997a). El impacto de esta pesquería, por lo general no es considerada en la evaluación de las poblaciones de camarón, como no lo fue en este trabajo ni en otros estudios realizados en el GT (Chávez 1974, 1979, Chávez \& Arreguín-Sánchez 1982, López-Rasgado 2003, García-Jiménez 2004, Gallardo-Berumen 2005, Ramos-Cruz et al. 2006, Cervantes-Hernández 2008). Debido a este problema, una fracción del valor de la tasa de mortalidad total (Z) que se ejerce sobre una cohorte a lo largo de su vida, no es correctamente estimada, por lo cual, la cohorte soporta una mortalidad total acumulada mayor a lo que los modelos suponen en alta mar (Gracia \& Vázquez-Bader 1999), que repercute en una baja de la producción total de las poblaciones de camarón. Al respecto, CervantesHernández et al. (2006) reportaron una reducción del 40\% en la captura total de camarón de alta mar en el GT entre 1983 y 2000, la cual fue correlacionada negativamente $(-0,5377, P=0,02)$ con las capturas totales obtenidas en los sistemas lagunares Mar Muerto, La Joya-Buenavista, Los Patos-Solo Dios, Carretas-Pereyra y ChantutoPanzacola. Gracia (1997a) señaló que el aumento del esfuerzo de pesca sobre los juveniles de camarón origina una sobrepesca del crecimiento, ya que se explota a los organismos antes de que se exprese la biomasa individual más alta, lo que ocurre en el ambiente marino, afectando los niveles de la captura global de la pesquería.

La sobrepesca del crecimiento es un proceso común en varias pesquerías de camarón tanto en el Pm como en el Golfo de México (Gracia 1995, Gracia 1997a, b, Gracia \& Vázquez-Bader 1997). El incremento excesivo del esfuerzo ribereño, aunado al esfuerzo de la pesca industrial, ocasiona serios problemas en las poblaciones naturales de camarón, por lo que es necesario considerar un balance entre éstos, con el fin de alcanzar una explotación óptima del recurso (Gracia \& Vázquez-Bader 1997). Dicho objetivo se encuentra lejos de los alcances de este trabajo, ya que sólo se consideró la explotación en alta mar. No obstante, para el caso de una pesquería secuencial como la del GT, es pertinente considerar los diferentes niveles de explotación que finalmente, repercutirán en el nivel del reclutamiento y la población reproductora (Gracia 1997b).

Otros factores complementarios que deben ser considerados para comprender la mayor intensidad de explotación sobre el camarón blanco y por qué en GT, 
ocurrió un cambio en la proporción de las capturas de ambas especies entre 1993 y 1997, se relacionan con la distribución espacial de estas especies, con respecto a la línea de costa y a la profundidad de pesca.

El camarón blanco L. vannamei, al igual que otras especies comúnmente conocidas como blancos, tienen una distribución batimétrica más somera en comparación a $F$. californiensis (considerado como camarones de color) (García \& Le Reste 1986, Gracia 1992). En el GT, ReynaCabrera \& Ramos-Cruz (1998) reportaron que el camarón blanco muestra un patrón de distribución espacial más cercano a las costas de Oaxaca y Chiapas en comparación con el camarón café. La captura del camarón blanco se realiza entre tres y 20 brazas de profundidad (5,5-36,4 $\mathrm{m})$; mientras que, la del camarón café, se desarrolla a profundidades de entre 15 y 20 brazas (27,3-36,4 m).

Considerando las características antes señaladas, la explotación de los reproductores de camarón blanco en comparación con el café, resulta en menores costos de inversión y tiempo de pesca. Esta es una de las razones que propició que la abundancia de los reproductores de camarón blanco, sea mermada rápidamente durante los tres primeros meses en las temporadas de pesca; mientras que, en este mismo lapso de tiempo, los reproductores de camarón café no están sujetos a la misma presión de explotación, debido a que su distribución espacial se encuentra más alejada a la línea de costa y su profundidad de pesca es mayor. Lo anterior pudo observarse indirectamente, a partir de la fecha en la cual la anomalía de los reproductores de ambas especies cambio de signo (de positivo a negativo), esto es, la flota de alta mar orientó por completo la presión de pesca hacia la población reproductora de camarón café, una vez ocurrido el cambio de signo de la anomalía de los reproductores de camarón blanco; sin embargo, antes de que esto ocurra, la población reproductora de camarón café obtuvo un período de tres meses bajo un régimen de explotación menor, en comparación con el que se ejerce sobre el camarón blanco al inicio de las temporadas de veda.

Los cambios anuales de la componente $C$ en términos de la edad de reclutamiento y los grupos de edad que conformaron a la población reproductora, sugieren que en el GT, entre 1993 y 1997, el sistema unificado vedas diseñado para todo el Pm, no cumple con los objetivos históricos y actuales para los cuales éstas fueron implementadas, esto es, la protección simultánea de los períodos de reproducción y de desove en las zonas de pesca de alta mar. En el GT, dichas temporadas protegieron básicamente al reclutamiento de ambas especies, el cual ayudó en parte a la población reproductora a incrementar la biomasa de las cohortes que coincidieron con dichas temporadas; no obstante, la protección de los reproductores fue mínima y no exitosa, ya que una vez que se abre la temporada de pesca, el esfuerzo pesquero causa una disminución notable en el número de reproductores antes de que éstos participen en el principal evento de reproducción, que origina el principal reclutamiento de la siguiente temporada.

La población reproductora remanente al final de cada temporada de pesca, comparativamente baja en ambas especies, en especial para el camarón blanco; es crítica para determinar el nivel del reclutamiento en la siguiente generación y por ende, el éxito de la siguiente temporada de pesca. Particularmente, si se considera la existencia de una relación parentela-progenie en los camarones Peneidos, modulada por factores ambientales (Gracia 1991, Cervantes-Hernández 1999). Varias especies de camarones Peneidos como P. merguiensis, $P$. semisulcatus, L. notialis y L. setiferus presentan una situación similar donde el principal reclutamiento es originado a partir de poblaciones reproductoras reducidas (Rothlisberg et al. 1985, Mathews et al. 1987, Lhome \& García 1984, Gracia 1989). Gracia (1996) indicó que las poblaciones de camarones Peneidos pueden soportar niveles de explotación que reduzcan la población reproductora hasta en un $20 \%$, sin afectar seriamente el reclutamiento; por debajo de este límite se origina la sobrepesca del reclutamiento, que repercute en los niveles de la captura global de la pesquería.

La estrategia más adecuada para la protección de las pesquerías de camarón en el GT, debe contemplar la protección de los reproductores, considerando la estimación del tamaño mínimo de éstos, que aseguren el máximo reclutamiento para la siguiente generación. Las temporadas de veda en el GT se aplican actualmente de marzo a septiembre (INP 2007a, 2007b), por lo tanto, el esquema de explotación para ambas especies de camarón, es el mismo que el reportado en este trabajo. Por ello, y con base en los resultados obtenidos, se propone para el GT, un corrimiento a dichos meses, para que las temporadas de veda sean de julio/junio a octubre y con ello, asegurar la protección de los reproductores y el crecimiento de los reclutas de ambas especies.

Adicionalmente, es importante considerar la regulación del esfuerzo pesquero, tanto en alta mar como en los sistemas lagunares del GT, para no sobrepasar los límites de éstos al final de las temporadas de pesca. La regulación del esfuerzo pesquero en los sistemas lagunares del GT es un punto particularmente importante ya que de acuerdo a la estrategia reproductiva del camarón, la protección de los estados juveniles puede tener mayor influencia para incrementar la fecundidad poblacional, 
que tan solo la protección de la población reproductora remanente en alta mar (Gracia 1995). Gracia \& VázquezBader (1997) sugieren que una estrategia de manejo basada en niveles de escape de organismos en cada una de las diferentes etapas explotadas, en combinación con reducciones de esfuerzo de pesca, cuotas de captura y vedas temporales, puede tener un alto impacto en la conservación del recurso y en los niveles de la producción pesquera. Sin embargo, para desarrollar una estrategia de este tipo, es necesario contar con una mayor información acerca de la dinámica poblacional de las especies de camarón y del esfuerzo pesquero en sus diferentes niveles. La información generada en este trabajo, puede servir como base para proponer la diferenciación entre temporadas de veda con respecto a todo el Pm y promover un ajuste para estructurar un planteamiento de vedas más acorde a los cambios anuales de la abundancia de reclutas y reproductores de ambas especies en el GT.

\section{Agradecimientos}

Los autores agradecen al Centro Regional de Investigación Pesquera de Salina Cruz, Oaxaca, México, por proveer la base de datos. A la Universidad del Mar de Puerto Ángel, por los recursos monetarios aportados a través del proyecto CRIP-UMAR:2IR043. Agradecemos los comentarios y sugerencias de los revisores anónimos.

\section{Literatura citada}

Cervantes-Hernández P. 1999. Relaciones stockreclutamiento del camarón Farfantepenaeus duorarum en el banco de Campeche. Tesis de Maestría, Instituto de Ciencias del Mar y Limnología, Universidad Nacional Autónoma de México, Distrito Federal, 37 pp.

Cervantes-Hernández P, S Ramos-Cruz \& A Gracia. 2006. Evaluación del estado de la pesquería de camarón en el Golfo de Tehuantepec. Hidrobiológica 16(3): 233-239.

Cervantes-Hernández P. 2008. Method to obtain indices of abundance in the population of brown shrimp from the Gulf of Tehuantepec, Oaxaca, México. Revista de Biología Marina y Oceanografía 43(1): 111-119.

Chávez EA. 1974. Estudio preliminar la época de entrada de postlarvas de camarón a las Lagunas Oriental y Occidental en el Sistema Lagunar Huave, Oaxaca. Informe Definitivo, 269 pp. Escuela Nacional de Ciencias Biológicas, Instituto Politécnico Nacional, México.

Chávez EA. 1979. Diagnosis de la pesquería del camarón del Golfo de Tehuantepec, Pacífico Sur de México. Anales del Instituto de Ciencias del Mar y Limnología, Universidad Nacional Autónoma de México 6(2): 15-44.

Chávez EA \& F Arreguín-Sánchez. 1982. Evaluación y diagnostico de la pesquería de camarón del Golfo de Tehuantepec. Informe Definitivo, 60 pp. Centro de
Investigación y Estudios Avanzados, Unidad Mérida, México, Instituto Politécnico Nacional, México.

Gallardo-Berumen MI. 2005. Análisis del sistema de vedas sobre la explotación del recurso camarón en el Golfo de Tehuantepec. Tesis de Licenciatura, Universidad del Mar, Puerto Ángel, Oaxaca, 48 pp.

García S \& L Le Reste. 1986. Ciclos vitales, dinámica, explotación y ordenación de las poblaciones de camarones peneidos costeros. FAO Documento Técnico de Pesca 203: 1-376.

García-Jiménez MA. 2004. Variación estacional del reclutamiento de camarón en la Pampona (Playa Bernal) en la Laguna Mar Muerto, Oaxaca-Chiapas, México. Tesis de Licenciatura, Universidad del Mar, Puerto Ángel, Oaxaca, 95 pp.

Gracia A. 1989. Ecología y pesquería del camarón blanco Penaeus setiferus en la Laguna de Términos-Sonda de Campeche. Tesis Doctoral, Facultad de Ciencias, Universidad Autónoma de México, Distrito Federal, 127 pp.

Gracia A. 1991. Spawning stock-recruitment relationship of white shrimp in the southwestern Gulf of Mexico. Transactions of the American Fisheries Society 120: 519-527.

Gracia A. 1992. Explotación y manejo del recurso camarón. Ciencia y Desarrollo 18(106): 82-95.

Gracia A. 1995. Impacto de la pesca artesanal sobre la producción de camarón rosado Penaeus Farfantepenaeus duorarum Burkenroad, 1939. Ciencias Marinas 21: 343-359.

Gracia A. 1996. White shrimp (Penaeus setiferus) recruitment overfishing. Marine and Freshwater Research 47: 59-65.

Gracia A. 1997a. Pesquería artesanal del camarón. En: Flores D, P Sánchez-Gil, JC Seijo \& F Arreguín-Sánchez (eds). Análisis y diagnóstico de los recursos pesqueros críticos del Golfo de México, pp. 173-184. Centro de Ecología, Pesquerías y Oceanografía del Golfo de México, Universidad Autónoma de Campeche, México.

Gracia A. 1997b. Simulated and actual effects of the brown shrimp, Penaeus aztecus, closure in Mexico. Marine Fisheries Review 59: 18-24.

Gracia A \& AR Vázquez-Bader. 1997. The effects of artisanal fisheries on penaeid shrimp stocks in the Gulf of México. En: Funk F, TJ Quinn II, J Heifetz, JN Ianelli, JE Powers, JF Schweigert, PJ Sullivan \& CI Zhang (eds). Proceedings of the International Symposium Fishery Stock Assessment Models for the $21^{\text {st }}$ Century. 127-144 pp. University of Alaska, Sea Grant College, Juneau, Alaska.

Gracia A \& AR Vázquez-Bader. 1999. Shrimp fisheries in the south Gulf of Mexico. Present and Future Management Alternatives. En: Kumpf H, D Steidinger \& K Sherman (eds). The Gulf of Mexico large Marine Ecosystem: Assessment, Sustainability, and Management, pp. 205-234. Blackwell Science, Berlin.

Instituto Nacional de la Pesca. 1997-1998. Sustentabilidad y Pesca Responsable en México, Evaluación y Manejo. Informe Definitivo, pp. 21-22. Secretaría de Medio Ambiente, Recursos Naturales y Pesca, México. 
Instituto Nacional de la Pesca (INP). 2000. Sustentabilidad y Pesca Responsable en México, Evaluación y Manejo, 691 pp. Secretaría de Medio Ambiente, Recursos Naturales y Pesca, México.

Instituto Nacional de la Pesca. 2004. Inicio de las temporadas de veda 2004 para la pesquería de camarón del Océano Pacifico Mexicano. Informe Definitivo, pp. 32-33. Secretaría de Medio Ambiente, Recursos Naturales y Pesca, México.

Instituto Nacional de la Pesca. 2007a. Resultado del análisis de las poblaciones de camarón del litoral del Pacífico para implementar la veda durante el 2007. Informe Definitivo, pp. 3-4. Secretaría de Medio Ambiente, Recursos Naturales y Pesca, México.

Instituto Nacional de la Pesca. 2007b. Resultados de los muestreos de las poblaciones de camarón, durante la veda del 2007 en el litoral del pacífico. Informe Definitivo, pp. 25-26. Secretaría de Medio Ambiente, Recursos Naturales y Pesca, México.

Kendall MG. 1984. Time Series, 542 pp. Oxford University Press, Nueva York.

López-Rasgado F. 2003. Evaluación de la eficiencia de la Red Renfro en el muestreo de camarón mediante experimentos de remoción en encierros en la Laguna Mar Muerto, Oaxaca-Chiapas, México. Tesis de Licenciatura, Universidad del Mar, Puerto Ángel, Oaxaca, 64 pp.

Lhome F \& S García. 1984. Biologie et exploitation de la crevette pénaeide au Sénegal. En: Gulland JA \& BJ Rothschild (eds). Penaeids shrimps-their biology and management, pp. 11-144. Fishing New Books, Farnham.

Magallón-Barajas F. 1987. The Pacific shrimp fishery of Mexico. CalCOFI Report 28: 43-52.

Mathews CP, M Al-Hossaini, AR Abdul Ghaffar \& M AlShousani. 1987. Assessment of short-lived stocks with special reference to Kuwait's shrimp fisheries: a contrast of the results obtained from traditional and recent size-based techniques. En: Pauly D \& GR Morgan (eds). Length based
Methods, ICLARM Conference Proceedings. 147-166 pp. Manila, Philipines.

Morales-Bojórquez E, J López-Martínez \& S HernándezVásquez. 2001. Modelo dinámico de captura y esfuerzo para el camarón café Farfantepenaeus californiensis (Holmes, 1900) del Golfo de California, México. Ciencias Marinas 27(1):105-124.

Pope J. 1972. An investigation of the accuracy of virtual population analysis using cohort analysis. International commercial Northwest Atlanta. Fish Research Bulletin 9: 65-74.

Ramos-Cruz S, B Sánchez-Meraz, F Carrasco-Ayuso \& P Cervantes-Hernández. 2006. Estimación de la tasa de mortalidad natural de Farfantepenaeus californiensis (Holmes 1900) y Litopenaeus vannamei (Boone 1931) en la zona costera del Golfo de Tehuantepec, México. Revista de Biología Marina y Oceanografía 41(2): 221-229.

Reyna-Cabrera IE \& S Ramos-Cruz. 1998. La pesquería de camarón de alta mar. En: Tapia-García M (ed). El Golfo de Tehuantepec: el ecosistema y sus recursos, pp. 163-178. Universidad Autónoma Metropolitana-Iztapalapa, México.

Rothlisberg PC, DJ Staples \& PJ Crocos. 1985. A review of the life history of the banana prawn, Penaeus merguiensis in the Gulf of Carpentaria. En: Rothlisberg PC, BJ Hill \& DJ Staples (eds). Second National Prawn Seminar, pp. 125136. Cleveland, Australia.

Rodríguez de la Cruz MC. 1976. Fundamentos y consideraciones para definir la temporada de veda en alta mar, para el camarón del Pacífico mexicano durante 1978. Informe Definitivo, 17 pp. Instituto Nacional de la Pesca. México.

Seber GAF. 1982. The estimation of animal abundance and related parameters, 654 pp. Macmillan Publishing Company, Nueva York.

Uriel E. 1995. Análisis de datos, series temporales y análisis multivariante, 433 pp. Universidad de Valencia, Madrid.

Recibido el 23 de mayo de 2007 y aprobado el 30 de abril de 2008 